\title{
Regulated Overexpression of Interleukin 11 in the Lung Use to Dissociate Development-dependent and -independent Phenotypes
}

Prabir Ray, ${ }^{*}$ Weiliang Tang, ${ }^{\star}$ Ping Wang, ${ }^{*}$ Robert Homer, ${ }^{\ddagger}$ Charles Kuhn III,

* Yale University School of Medicine, Section of Pulmonary and Critical Care Medicine, Department of Internal Medicine, New Haven, Connecticut 06520-8057; ${ }^{\ddagger}$ ale University School of Medicine, Department of Pathology, New Haven, Connecticut 06520-8023; ${ }^{\S}$ Connecticut VA Hospital, Pathology and Laboratory Medicine Service, West Haven, Connecticut 06516; "Brown University School of Medicine, Memorial Hospital of Rhode Island, Department of Pathology, Pawtucket, Rhode Island 02860; and "Yale University School of Medicine, Section of Immunobiology and Howard Hughes Medical Institute, New Haven, Connecticut 06520-8057

\begin{abstract}
Standard overexpression transgenic approaches are limited in their ability to model waxing and waning diseases and frequently superimpose development-dependent and -independent phenotypic manifestations. We used the clara cell $10-\mathrm{kD}$ protein (CC10) promoter and the reverse tetracycline transactivator (rtTA) to create a lung-specific, externally regulatable, overexpression transgenic system and used this system to express human interleukin 11 (IL-11) in respiratory structures. Gene induction could be achieved in utero, in neonates and in adult animals. Moreover, gene expression could be turned off by removal of the inducing stimulus. When gene activation was initiated in utero and continued into adulthood, subepithelial airway fibrosis, peribronchiolar mononuclear nodules, and alveolar enlargement (emphysema) were noted. Induction in the mature lung caused airway remodeling and peribronchiolar nodules, but alveolar enlargement was not appreciated. In contrast, induction in utero and during the first $\mathbf{1 4} \mathbf{d}$ of life caused alveolar enlargement without airway remodeling or peribronchiolar nodules. Thus, IL-11 overexpression causes abnormalities that are dependent (large alveoli) and independent (airway remodeling, peribronchiolar nodules) of lung growth and development, and the CC10-rtTA system can be used to differentiate among these effector functions. The CC10-rtTA transgenic system can be used to model waxing and waning, childhood and growth and development-related biologic processes with enhanced fidelity. (J. Clin. Invest. 1997. 100: 2501-2511.) Key words: emphysema - airway fibrosis • asthma $\bullet$ inducible transgene expression
\end{abstract}

\section{Introduction}

Organ-specific overexpression transgenic modeling uses tissue-specific promoters to drive the expression of the gene(s) of interest. This approach has provided remarkable insights into

Address correspondence to Jack A. Elias, M.D., Yale University School of Medicine, Section of Pulmonary and Critical Care Medicine, Department of Internal Medicine, 333 Cedar Street, LCI 105, New Haven, CT 06520-8057. Phone: 203-785-4163; FAX: 203-7853826; E-mail: jack.elias@yale.edu

Received for publication 3 June 1997 and accepted in revised form 29 August 1997.

The Journal of Clinical Investigation

Volume 100, Number 10, November 1997, 2501-2511

http://www.jci.org the biologic effector functions and interactions of a large number of important proteins. However, this approach is not without important limitations. Many occur because the promoters that are used often initiate gene expression in utero and drive gene expression in a largely constitutive fashion thereafter. This confounds phenotypic interpretation by superimposing growth and/or development-related abnormalities on the abnormalities that would otherwise be caused by the gene product in an adult animal. It may also generate phenotypes that are accurate representations of the effects of chronically expressed proteins, but are not representative of the effects of these proteins when they are expressed in an intermittent fashion. Thus, overexpression transgenic approaches have a limited ability to model waxing and waning disease processes and differentiate the effects of genes that are dependent on and independent of organ growth and development.

In an attempt to address the limitations of overexpression transgenic modeling, several investigators have developed transgenic systems in which gene expression can be externally regulated. Early attempts focused on a variety of approaches including the use of steroid-inducible and metallothioneinbased $(1,2)$ promoter systems. More recent approaches have used tet-regulatory systems based on wild-type and mutated tetracycline transactivator fusion proteins (3-5). Subsequent studies have demonstrated that tet-based systems can be externally regulated after direct injection into cardiac tissue (6), transfection into mesangial cells that are subsequently injected into visceral organs (7), and microinjection using standard transgenic technology (8-11). To date, however, these approaches have not been shown to selectively activate transgenes in utero, in neonates, and in adult animals, and have not been demonstrated to differentiate growth and developmentdependent and -independent phenotypic abnormalities.

The lung is an outstanding system in which to evaluate the utility of externally regulatable overexpression transgenic systems. It is affected frequently by chronic waxing and waning inflammatory disorders of the airway such as asthma. In addition, early childhood events, such as severe viral infections, are increasingly appreciated to be associated with and potentially involved in the pathogenesis of the asthmatic diathesis (1214). Overexpression modeling is being used to model asthma (15-17) and other waxing and waning inflammatory respiratory disorders $(18,19)$. These studies have most frequently used the clara cell $10-\mathrm{kD}$ protein promoter $(\mathrm{CC} 10)^{1}$ and the surfactant apoprotein-C promoter to target genes to the air-

1. Abbreviations used in this paper: $\mathrm{BAL}$, bronchoalveolar lavage; CC10, clara cell 10-kD protein; CMV, cytomegalovirus; dox, doxycycline; hGH, human growth hormone; pc, postcoitus; rtTA, reverse tetracycline transactivator; tet- $\mathrm{O}$, tetracycline operator. 
way and alveolus, respectively. However, these promoters are activated in utero during times of important respiratory morphogenesis $(20,21)$, setting the stage for targeted transgenes to alter lung development (21). The degree to which development-dependent and -independent abnormalities are being evaluated in these models has not been assessed adequately.

To characterize the merits of externally regulatable overexpression transgenic approaches and to facilitate the modeling of pulmonary disorders, we developed a lung-specific externally regulatable overexpression system and used this system to express human IL-11 for varying periods of time before and after birth. IL-11 was chosen because our previous studies demonstrated that it is produced by lung fibroblasts and epithelial cells in response to cytokines, histamine, eosinophil major basic protein, and respiratory tropic viruses (22-26) and is found in the nasal secretions of children with upper respiratory tract infections (26). The phenotypes of animals with regulated transgene expression were compared with each other and to the phenotype of traditional transgenic animals in which IL-11 was expressed under the influence of the same promoter without this regulatable feature. These studies demonstrate that the pulmonary phenotype of traditional IL-11 overexpression mice is a mixture of development-dependent and -independent manifestations. They also demonstrate that the CC10reverse tetracycline transactivator (rtTA) system can be used to selectively activate transgenes in utero, in neonates, and in adult animals, and can thus be used to differentiate development-dependent and -independent phenotypes and define crucial temporal windows during development that influence phenotype acquisition.

\section{Methods}

\section{Production of transgenic mice}

Transgenic mice were generated in which IL-11 was expressed in a lung-specific fashion using genetic constructs that did and did not allow for external regulation of transgene expression. The constructs that were used are illustrated in Fig. 1. Both used the $\mathrm{CC} 10$ promoter and took advantage of the fact that the murine respiratory epithelium contains $50-60 \%$ clara cells (27).

\section{CC10-rtTA-hIL-11 mice}

The externally regulatable transgenic system was based on the generation of animals with two transgenic constructs. The CC10-rtTA construct contained the $\mathrm{CC} 10$ promoter, the rtTA, and human growth hormone $(\mathrm{hGH})$ intronic, with its nuclear localization sequence and polyadenylation sequences. The rtTA is a fusion protein made up of a mutated tetracycline repressor and the herpes virus VP-16 transactivator (5). The tet-O-CMV-hIL-11 construct contained a polymeric tetracycline operator (tet-O), minimal cytomegalovirus (CMV) promoter, human IL-11 cDNA, and hGH intronic and polyadenylation signals. In this system the $\mathrm{CC} 10$ promoter should direct the expression of rtTA to the lung. In the presence of doxycycline (dox), rtTA is able to bind in trans to the tet-O, and the VP-16 transactivator activates IL-11 gene transcription. In the absence of dox, rtTA binding does not occur and transgene transcription is not activated.

Preparation of the CC10-rtTA construct. A 2.3-kb HindIII fragment containing the rat $\mathrm{CC} 10$ promoter was obtained from construct pCC10-CAT (a gift from Dr. J. Whitsett and Dr. B. Stripp, Cincinnati, $\mathrm{OH})(28)$. It was subsequently serially ligated to: $(a)$ a $1.0-\mathrm{kb}$ HindIII/BamHI fragment of plasmid 172.1 neo (a gift from Dr. M. Gossen and Dr. H. Bujard, University of Heidelberg, Heidelberg, Germany) (5) containing the rtTA gene; and (b) a 2.1-kb BamHI fragment containing the intronic and polyadenylation sequences of the hGH gene from plasmid p1017 (a gift from Roger M. Perlmutter, University of Washington, Seattle, WA). The ligated fragments was then cloned into the Hind III site in plasmid pBluescript II $\left(\mathrm{KS}^{+}\right)$ (Stratagene, La Jolla, CA). A 5.4-kb fragment containing the CC10rtTA-hGH minigene was purified and used for microinjection.

Preparation of the tet-O-CMV-hIL-11 construct. Construct pUHC13-3 was obtained from Dr. Gossen and Dr. Bujard (5) and a 0.49-kb $\mathrm{XhoI} / \mathrm{ClaI}$ fragment containing heptamerized tet-O sequences linked to the CMV minimal promoter was isolated. This fragment was then ligated to hGH intronic and polyadenylation sequences and a $0.7-\mathrm{kb}$ human IL-11 cDNA which had been digested previously with SmaI to remove its $3^{\prime}$ untranslated region. The entire DNA fragment was then cloned into the XhoI/BamHI site of pBluescript II $\left(\mathrm{KS}^{+}\right)$(Stratagene). The tet-O-CMV-hIL-11 DNA fragment was then isolated by digestion with $\mathrm{BssH} 2$, purified, and used for microinjection.

All constructs were checked for correct orientation of the inserts by restriction enzyme digestion and junction sequences were confirmed by sequencing. Both constructs were purified, linearized, separated by electrophoresis through agarose, and isolated by electroelution into dialysis tubing. The DNA fragments were then purified through Elutip-D columns following the manufacturer's instructions
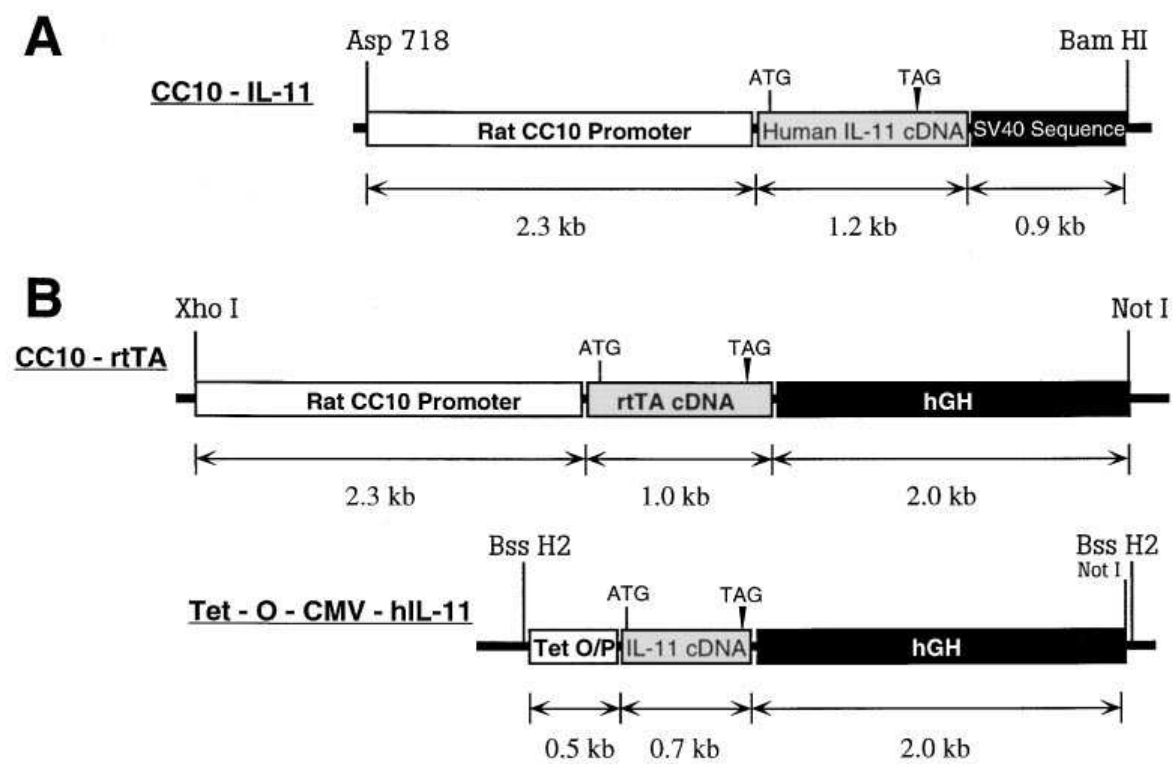

Figure 1. Genetic constructs used in the generation of the IL-11 overexpression transgenic mice. The traditional overexpression system $(A)$ used the rat $\mathrm{CC} 10$ promoter, IL-11 cDNA, and SV-40 intronic and polyadenylation sequences. The inducible system $(B)$ required two genetic constructs. The CC10-rtTA construct used the rat $\mathrm{CC} 10$ promoter, rtTA cDNA, and hGH intronic and polyadenylation sequences. The tet-O-CMV-hIL-11 construct used the tet-O, IL-11 cDNA, and hGH intronic and polyadenylation sequences. 
(Schleicher \& Schuell, Inc., Keene, NH) and dialyzed against injection buffer $(0.5 \mathrm{mM}$ Tris- $\mathrm{HCl} / 25 \mathrm{mM}$ EDTA, $\mathrm{pH} 7.5)$. Transgenic mice were prepared in [CBA $\times \mathrm{C} 57 \mathrm{BL} / 6] \mathrm{F}_{2}$ eggs by mixing and simultaneously injecting the constructs into pronuclei as described previously $(16,17)$.

\section{CC10-IL-11 mice}

These traditional lung-specific IL-11 overexpression mice used a genetic construct containing the CC10 promoter, IL-11 cDNA, and SV40 intronic and polyadenylation sequences. The generation of these animals has been described previously by this laboratory (16).

\section{Documentation of transgenic status}

The presence or absence of the transgenes were initially evaluated using Southern blot analysis and later by PCR.

Southern blotting. Mouse tail biopsy DNA was digested with BamH1 and the resulting fragments were separated by electrophoresis using a $1 \%$ agarose gel at $2 \mathrm{~V} / \mathrm{cm}$ in Tris-borate buffer. The resolved fragments were then transferred to a nylon membrane and hybridization was accomplished using an overnight incubation in a buffer containing $6 \times \operatorname{SSPE}\left(3 \mathrm{M} \mathrm{NaCl}, 0.2 \mathrm{M} \mathrm{NaH}_{2} \mathrm{PO}_{4} \cdot \mathrm{H}_{2} \mathrm{O}\right.$, and $0.02 \mathrm{M} \mathrm{Na}_{2}$ EDTA), $0.5 \%$ SDS, $50 \%$ formamide, $50 \mu \mathrm{g} / \mathrm{ml}$ salmon sperm DNA, and labeled rtTA or IL- $11 \mathrm{cDNA}$ probes $\left(5 \times 10^{6} \mathrm{cpm} / \mathrm{ml}\right.$ of hybridization buffer). The rtTA probe was a 1-kb EcoR1/BamH1 fragment from plasmid 172.1. The IL-11 cDNA probe was a $1.2-\mathrm{kb}$ cDNA fragment. After hybridization, the blots were washed twice for $15 \mathrm{~min}$ at room temperature in $6 \times \mathrm{SSPE} / 0.2 \%$ SDS and then twice for $15 \mathrm{~min}$ each at $55^{\circ} \mathrm{C}$ in $0.2 \times \mathrm{SSPE} / 1.0 \% \mathrm{SDS}$.

$P C R$. PCR for rtTA and IL-11 were performed using the following upper and lower primers and incubation conditions. rtTA PCR primers and conditions were: upper primer: 5'-GTCGCTAAAGAAGAAAGGGAAACAC-3'; lower primer: 5'-TTCCAAGGGCATCGGTAAACATCTG-3'; PCR conditions: 1 cycle of $95^{\circ} \mathrm{C}$ for $5 \mathrm{~min}$, 35 cycles of $95^{\circ} \mathrm{C}$ for $1 \mathrm{~min}, 59^{\circ} \mathrm{C}$ for $1 \mathrm{~min}$, and $72^{\circ} \mathrm{C}$ for $2 \mathrm{~min}$. IL- 11 PCR primers and conditions were: upper primer: $5^{\prime}$-CGACTGGACCGGCTGCTGC-3'; lower primer: 5'-CTAACTAGGGGGAGATAATGGCGGGGGGA-3'; PCR conditions: 30 cycles were performed. Each cycle was heated at $95^{\circ} \mathrm{C}$ for $1 \mathrm{~min}$, annealed at $63^{\circ} \mathrm{C}$ for $1 \mathrm{~min}$, and elongated at $70^{\circ} \mathrm{C}$ for $2 \mathrm{~min}$.

All CC10-rtTA-hIL-11 lineage animals were evaluated for the presence of both transgenes. All CC10-IL-11 lineage animals were evaluated for IL-11 only.

\section{dox water administration}

All inducible transgenic mice were maintained on normal water until the point in time when transgene activation was desired. At that time, dox $(0.5 \mathrm{mg} / \mathrm{ml})$ was administered using aluminum foil wrapped water bottles to prevent light-induced dox breakdown.

\section{Bronchoalveolar lavage (BAL) and quantification of \\ IL-11 levels}

Mice were killed via cervical dislocation, a median sternotomy was performed, blood was obtained via right heart puncture and aspiration, and serum was prepared. The trachea was then isolated via blunt dissection, and small caliber tubing was inserted and secured in the airway. Three successive washes of $0.75 \mathrm{ml}$ of PBS with $0.1 \%$ BSA were then instilled and gently aspirated. Each BAL aliquot was centrifuged and the supernatants were harvested and stored individually at $-70^{\circ} \mathrm{C}$. The levels of IL-11 in the BAL fluid and serum were quantitated immunologically via ELISA. The ELISA was performed as previously described by our laboratory $(23,24)$ using antibodies $11 \mathrm{~h} 3 /$ 15.6.1 and 11h3/19.6.1 provided by Dr. Edward Alderman (Genetics Institute, Cambridge, MA).

\section{Northern analysis}

Total cellular RNA from a variety of mouse tissues was obtained using guanidine isothiocyanate extraction and formaldehyde-agarose gel electrophoresis as described previously (16). IL-11 gene expres- sion was assessed by probing with ${ }^{32} \mathrm{P}-$ labeled IL-11 cDNA. Equality of sample loading and efficiency of transfer were assessed via ethidium bromide staining.

\section{Histologic evaluation}

Animals were killed via cervical dislocation and median sternotomies were performed. The heart and lungs were then removed en bloc and inflated to pressure $(25 \mathrm{~cm})$ with neutral buffered $10 \%$ formalin, fixed overnight in $10 \%$ formalin, embedded in paraffin, and sectioned and stained. Hematoxylin and eosin and Mallory's trichrome stains were performed.

\section{Lung organ cultures}

Fetal lung organ cultures were performed using modifications of the techniques of Gross and Wilson (29). The lungs from 14-19-d-old fetuses were isolated and the individual lobes were dissected and cultured separately. Equal fragments (by weight) of pulmonary tissue were placed in 35-mm plastic tissue culture dishes in Waymouth's MB $752 / 1$ medium supplemented with penicillin, streptomycin, and $1 \%$ fetal bovine serum. Scratches were made on the culture dishes to ensure the adherence of the explants. They were then incubated in 5\% $\mathrm{CO}_{2}$ at $37^{\circ} \mathrm{C}$. For the first $1.5 \mathrm{~h}$ they were in stationary cultures and for the remainder of the time they were incubated with gentle rocking. The supernatants surrounding the explants were removed at 24-h intervals and their IL-11 content was assessed by ELISA as described.

\section{Morphometric analysis}

Air space size was estimated from the mean chord length of the airspace (30). This measurement is similar to the mean linear intercept, a standard measure of air space size, but has the advantage that it is independent of alveolar septal thickness. Sections were prepared as described above. To obtain images at random for analysis, each glass slide was placed on a printed rectangular grid and a series of dots placed on the cover glass at the intersection of the grid lines, i.e., at $\sim 1$-mm intervals. Fields as close as possible to each dot were acquired by systemically scanning at $2-\mathrm{mm}$ intervals. Fields containing identifiable artifacts or nonalveolated structures such as bronchovascular bundles or pleura were discarded.

A minimum of 10 fields from each mouse lung was acquired into a Macintosh computer through a framegrabber board. Images were acquired in 8-bit grayscale at a final magnification of 1.5 or 2 pixels per micron. The images were analyzed on a Macintosh computer using the public domain NIH Image program written by Wayne Rasband at the U.S. National Institutes of Health and available at http:// rsb.info.nih.gov/nih-image. Images were manually thresholded, then smoothed, and inverted. The image was then subject to sequential logical image match and operations with a horizontal and then vertical grid. At least 200 measurements per field were made in transgene positive animals and 400 measurements per field were made in the transgene negative animals. The length of the lines overlying air space air was averaged as the mean chord length. Standard deviation was calculated using techniques that take animal to animal and field to field variations into account $(31,32)$. At least four animals were studied at each time point in the presence and absence of dox water. Chord length increases with alveolar enlargement.

\section{Statistical analysis}

Values are expressed as means \pm SEM. Unless otherwise stated, group means were compared by ANOVA with Scheffe's procedure posthoc analysis using StatView software for the Macintosh.

\section{Results}

Generation of transgenic mice. To generate transgenic mice in which IL-11 was overproduced in a lung-specific and externally regulatable fashion, the two necessary genetic constructs 
were produced and simultaneously microinjected. From these microinjections, 10 transgenic animals were obtained. One animal had the CC10-rtTA construct only. The other nine contained both of the desired genetic constructs. These founder animals were bred with C57 BL/6 mice and the transgene status of the offspring was similarly analyzed. This analysis demonstrated that both transgenes consistently traveled with one another and passed on to the offspring of the dual transgene positive founder animals in a Mendelian fashion. This suggests that the constructs inserted into the host genome in an identical and/or similar location. Of the dual founder lines, three did not manifest inducible IL-11 production. Two of the others were chosen for detailed analysis based on their low level of basal IL-11 production and significant response to dox water. Since their phenotypes were essentially identical, they are described below in a unified fashion.

Induction of IL-11 expression. To determine if the CC10rtTA-hIL-11 system was functioning properly, adult ( $\geq 2$ mo of age) dual transgene positive and transgene negative mice were maintained on normal water or water supplemented with dox $(0.5 \mathrm{mg} / \mathrm{ml})$ for varying periods of time. Their lungs were then removed, BAL was performed, and the levels of BAL fluid IL-11 were evaluated by ELISA. In accord with the fundamental assumptions of the inducible system, human IL-11 protein was unable to be detected in the BAL fluid of trans- gene negative mice drinking normal water or dox water, and IL-11 was unable to be detected, or was marginally detected, in the BAL fluid of dual transgene positive mice drinking normal water (Fig. 2 and data not shown). In contrast, human IL-11 protein was readily detected in the BAL fluid of dual transgene positive mice drinking dox water (Fig. $2 A$ ). This induction could be appreciated after as little as $24-48 \mathrm{~h}$ and appeared to plateau $\sim 4-6 \mathrm{~d}$ after the addition of dox to the animal's water supply. At all time points, IL-11 was unable to be detected in the serum of these animals (data not shown). In accord with our findings with IL-11 protein, human IL-11 mRNA was unable to be detected in the lungs of transgene negative animals on normal or dox water, and human IL-11 mRNA was barely detected or undetectable in the lungs of dual transgene positive animals drinking normal water (Fig. 2 and data not shown). In contrast, human IL-11 mRNA was readily detected in the lungs of dual transgene positive animals within $24 \mathrm{~h}$ of the addition of dox to their water supply (Fig. $2 \mathrm{~B}$ ). These studies demonstrate that, in CC10-rtTA-hIL-11 mice, very modest levels of IL-11 are produced at baseline and that the IL-11 transgene is readily induced by dox water.

Cessation of IL-11 production and gene expression. The rtTA system should allow genes to be turned on and off. Thus studies were undertaken to determine if removal of dox from a dual transgene positive animal's water supply resulted in the

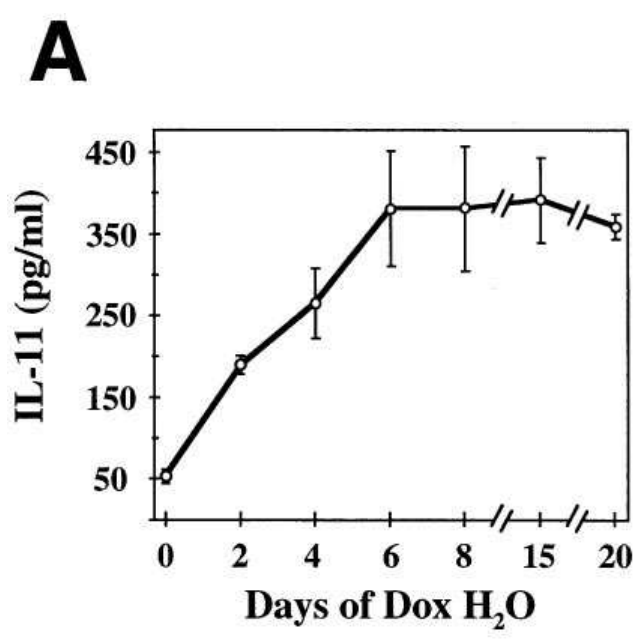

C

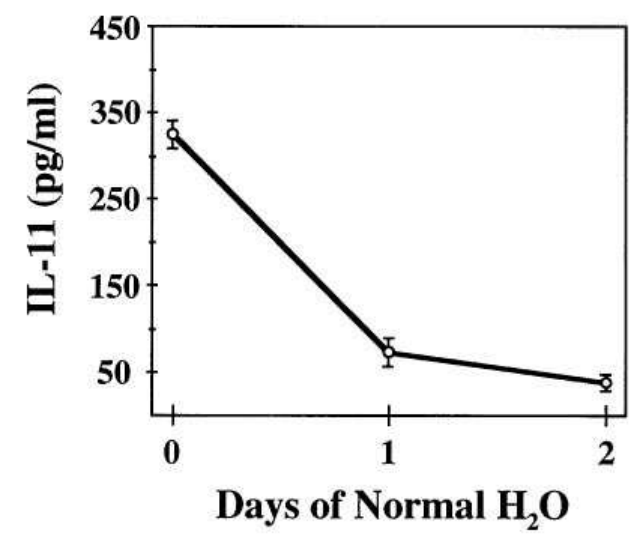

Days of Dox $\mathrm{H}_{2} \mathrm{O}$
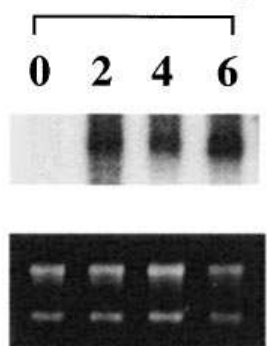

Ethidium

Bromide
D

\section{Days of Normal $\mathrm{H}_{2} \mathrm{O}$

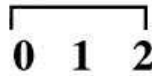

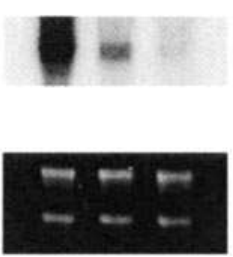

IL-11

Ethidium Bromide
Figure 2. Kinetics of induction and cessation of IL-11 production in CC10rtTA-hIL-11 mice. In $A$ and $B$, dual transgene positive mice were grown to adulthood on normal water and then dox $(0.5$ $\mathrm{mg} / \mathrm{ml}$ ) was added to their water supply. The levels of BAL IL-11 protein $(A)$ and pulmonary IL-11 mRNA $(B)$ were assessed at intervals before and after the addition of dox. In $C$ and $D$, dual transgene positive mice received dox water for $1 \mathrm{wk}$ and then the dox was removed. The levels of BAL IL-11 protein $(C)$ and pulmonary IL-11 mRNA $(D)$ were assessed at intervals before and after dox removal. $B$ and $D$ are representative experiments of $n=3$. $A$ and $C$ represent the mean \pm SEM of triplicate determinations at each time point. 
cessation of transgene protein production and gene expression. In these experiments, dual transgene positive adult animals were maintained on dox water for $1 \mathrm{wk}$ and were then placed on normal water. The levels of IL-11 protein and mRNA in their BAL fluid and lungs, respectively, were assessed at intervals thereafter. As shown in Fig. 2, $C$ and $D$, IL-11 production in CC10-rtTA-hIL-11 dual transgene positive animals was dox-dependent with IL-11 gene expression and BAL IL-11 protein levels decreasing significantly ( $\geq 80 \%$ ) within $24 \mathrm{~h}$ of dox removal. In addition, IL-11 gene expression and protein production could be turned on, off, and on in these animals without any overt dampening of the responsiveness of this system (data not shown).

Organ specificity of dox-induced IL-11 expression. This externally regulatable overexpression system is designed to provide lung-specific transgene expression. To determine if the desired respiratory targeting had been accomplished, dual transgenic animals were maintained on dox water for $1 \mathrm{wk}$ and the levels of human IL-11 in their BAL fluid and serum and the levels of human IL-11 mRNA in pulmonary and a variety of other tissues were compared. As noted above, IL-11 protein was readily detected in BAL fluid. However, it was not able to be detected in significant quantities in serum (data not shown). In addition, IL-11 mRNA was readily detected in lung tissue but not in RNA from a variety of other structures including heart, intestine, kidney, liver, muscle, pancreas, skin, spleen, testis, and uterus (Fig. 3 and data not shown). Thus, the CC10rtTA system successfully targeted IL-11 to the lung.

Activation of the IL-11 transgene in utero and in neonatal animals. A desirable feature of an externally regulatable overexpression transgenic system designed for investigations of organ growth and development is the ability to activate gene expression before birth, just after birth, or in adult animals. The data presented above demonstrate that dox activates IL-11 in adult animals. To determine if dox was able to activate gene expression in utero, dual transgene positive male mice and wild-type female mice were allowed to mate and the resulting gravid female mice were given normal water or dox water. The levels of IL-11 gene expression in the lungs of the resulting

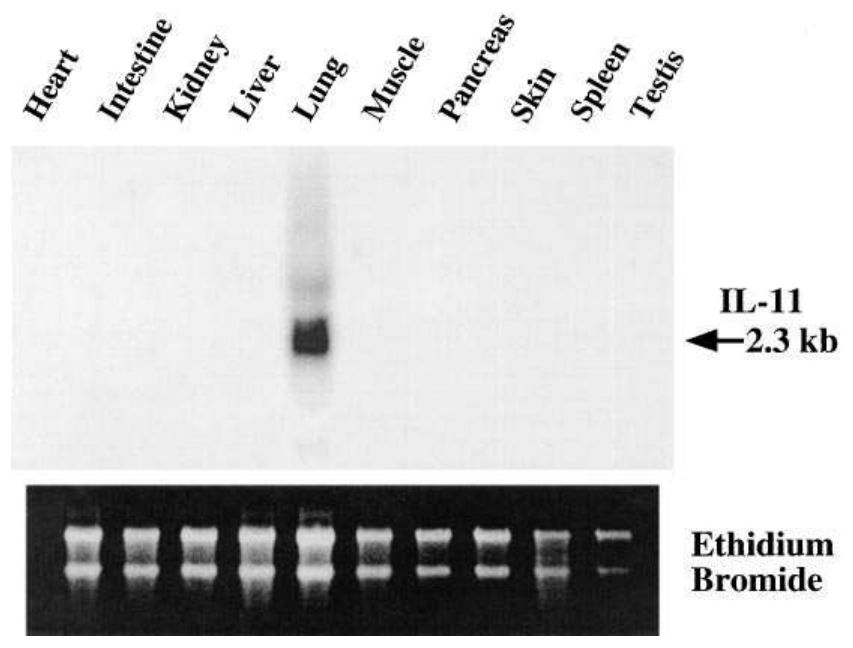

Figure 3. Organ specificity of transgene activation in CC10-rtTAhIL-11 mice. Dual transgene positive mice were maintained on dox water for $1 \mathrm{wk}$. The levels of IL-11 gene expression in the noted structures were then assessed by Northern analysis.

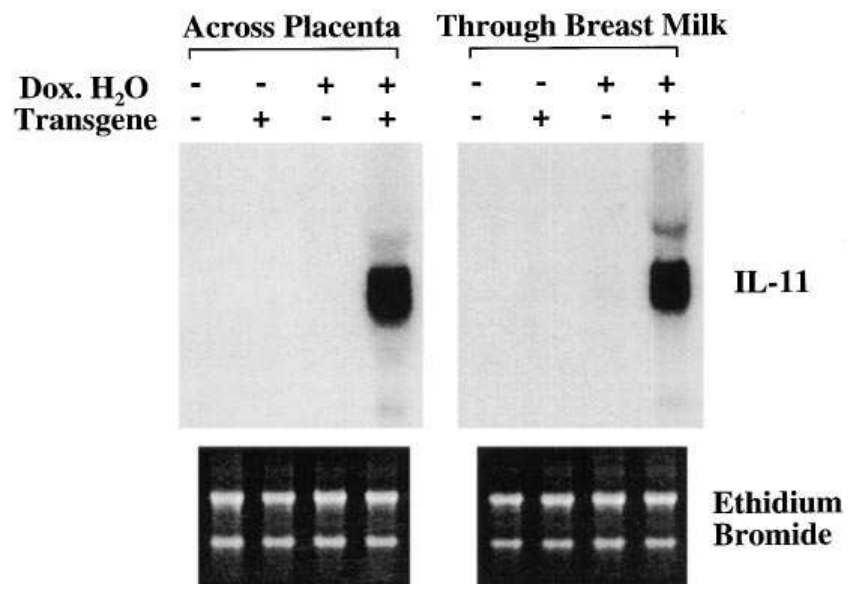

Figure 4. Activation of the IL-11 transgene in utero and in neonatal animals. Dual transgene positive male mice were crossed with transgene negative female mice. In the left panel (Across Placenta) the gravid female mice received normal water $(-)$ or dox water $(+)$ and the transgene status and levels of pulmonary IL-11 mRNA of the pups were assessed at birth. In the right panel (Through Breast Milk) dual transgene positive male mice were similarly mated with wildtype female mice and allowed to carry to term. The suckling mothers were then given either normal water $(-)$ or dox water $(+)$ and the lungs of their offspring were assessed at 2 wk of age. Comparisons were made of the levels of IL-11 mRNA in the lungs of transgene negative ( $(-)$ and dual transgene positive $(+)$ animals. The ethidium bromide loading controls are seen below each panel.

pups were assessed at birth. As can be seen in Fig. 4, IL-11 mRNA was unable to be detected in the lungs of transgene negative animals receiving normal water or dox water, and IL-11 gene expression was unable to be detected or was barely detected in the lungs of dual transgene positive animals whose mothers received normal water. In contrast, impressive levels of IL-11 gene expression were detected in the lungs of dual transgene positive pups whose mothers received dox water. This demonstrates that dox is passed through the placenta in quantities that are sufficient to activate gene expression in utero.

To determine if IL-11 gene expression was able to be activated in neonates, similar matings of dual transgene positive males and transgene negative females were undertaken and gravid female mice receiving normal water were allowed to carry to term. After their pups were born, some mothers were continued on normal water while others received dox water. 2 wk later, the IL-11 gene expression in the lungs of the pups was assessed. IL-11 mRNA was unable to be detected in the lungs of transgene negative pups whose mothers received normal water or dox water. IL-11 gene expression was also undetectable, or barely detectable, in the lungs of dual transgene positive pups whose mothers received normal water. In contrast, IL-11 gene expression was readily detected in the lungs of dual transgene positive pups whose mothers received dox water (Fig. 4). Thus, sufficient amounts of dox are transferred from lactating mothers to infants to activate rtTA-regulated genes in dual transgene positive offspring. When viewed in combination, these studies demonstrate that the CC10-rtTAbased system can be used to activate transgene expression in utero, in neonates, or in adult animals. 

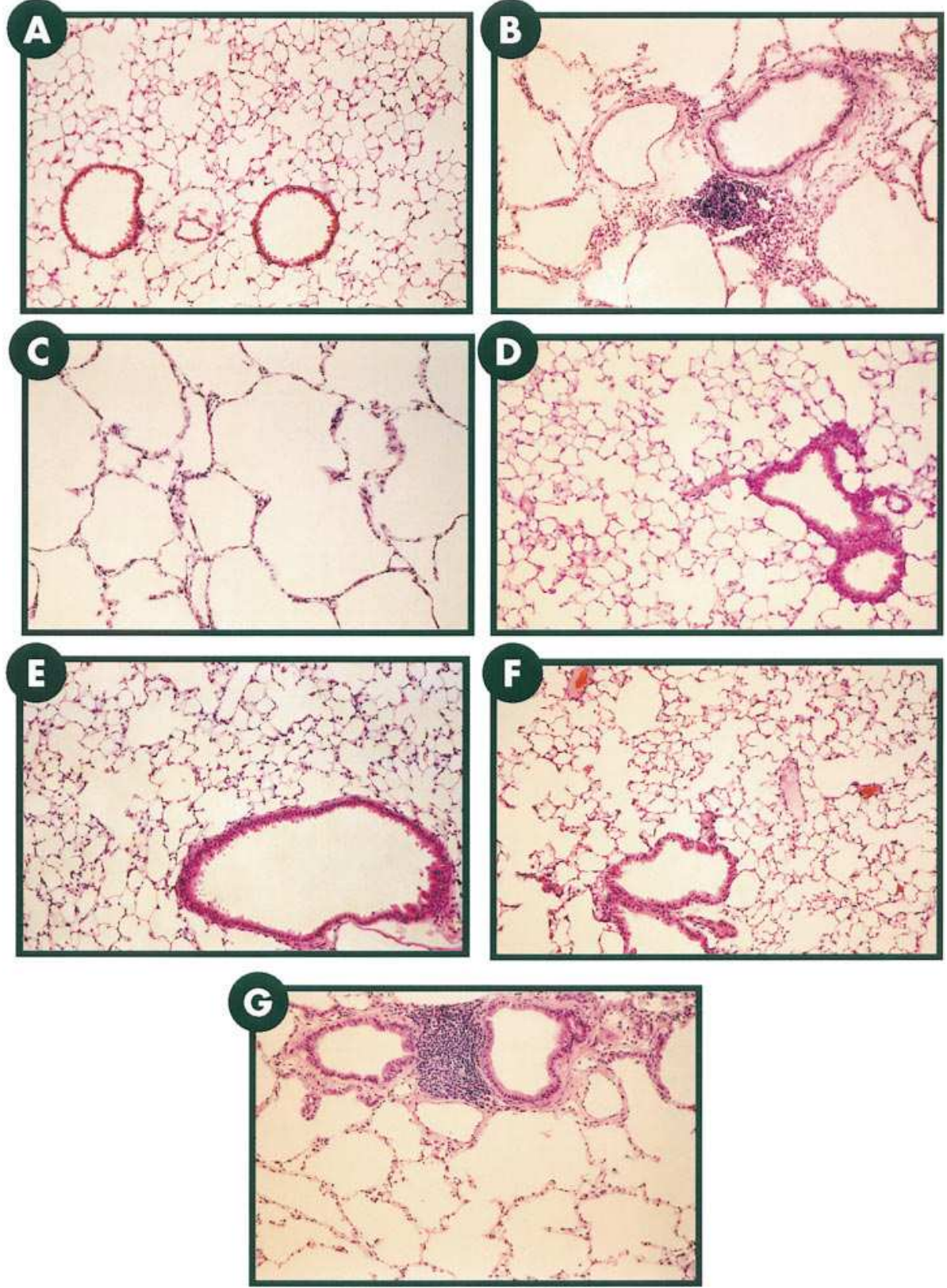

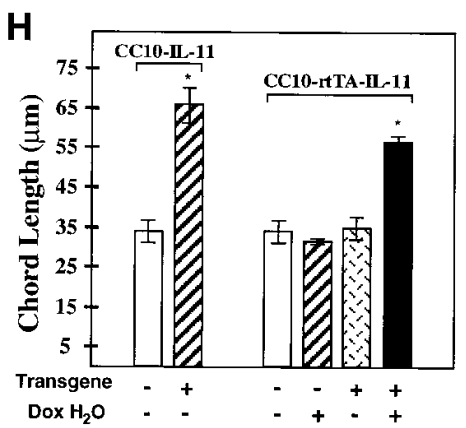

Figure 5. Comparison of the airway and alveolar phenotype of CC10-IL-11 and CC10-rtTA-hIL-11 animals when transgene activation was initiated in utero and maintained for 2 mo. $A$ illustrates the normal pulmonary anatomy of transgene negative control mice. $B$ illustrates the peribronchial nodules and subepithelial fibrosis seen in the airways of the CC10IL-11 mice. $C$ illustrates the large peripheral alveoli seen in CC10-IL-11 mice. $D$ and $E$ illustrate the normal pulmonary anatomy of transgene negative mice on normal water and dox water, respectively. $F$ illustrates the normal pulmonary anatomy of dual transgene positive CC10-rtTA-hIL-11 mice on normal water. $G$ illustrates the airway remodeling, peribronchiolar nodules, and enlarged air spaces seen in the CC10-rtTA-hIL-11 mice when the IL-11 transgene was activated in utero and expressed chronically thereafter. $H$ illustrates the morphometric features (chord length) of the alveolar portions of the lungs from transgene positive and transgene negative animals from both lines. ${ }^{*} P<0.02$ comparing $\mathrm{CC} 10$ IL-11 transgene positive and negative littermates, and dual transgene positive CC10-rtTA-hIL-11 mice on dox water to transgene negative littermates on normal or dox water or dual transgene positive littermate mice on normal water. Original magnification for all photomicrographs, $\times 39.2$.
Characterization of kinetics of CC10 activation. To be able to accurately compare the traditional CC10-IL-11 and inducible CC10-rtTA-hIL-11 mice, studies were undertaken to accurately define the kinetics of IL-11 production in the CC10-IL-11 animals. As described previously (16), IL-11 mRNA and/or protein were easily appreciated in the lungs and/or BAL fluid of 2-d-old to 6-mo-old transgene positive mice, but not their transgene negative littermates (data not shown). To determine if CC10 was activated in utero, lung organ cultures were prepared from transgene positive mice 14-19 d postcoitus (pc) and the IL-11 content of the supernatants of these cultures was assessed by ELISA. In keeping with prior reports $(20,21)$, IL-11 could not be detected in supernatants from cultures of day 15 or $16 \mathrm{pc}$ lungs, but was readily detected in supernatants from days 17-18 pe lungs (data not shown). Thus, in traditional CC10-IL-11 transgenic mice, CC10 promoter activation can be detected by days $17-18$ of gestation and continues for at least 6 mo thereafter.

Comparison of phenotypes of CC10-rtTA-hIL-11 and CC10-IL-11 mice. Knowledge of the kinetics of IL-11 expression in the traditional transgenic animals was then used to allow us to compare the phenotype of adult (2-3-mo-old) CC10IL-11 mice and CC10-rtTA-hIL-11 mice in which IL-11 was expressed for similar periods of time. The CC10-IL-11 transgene positive animals had three major pathologic alterations. As previously noted (16), their airway alterations included peribronchiolar mononuclear cell predominant nodules and subepithelial fibrosis (Fig. $5 \mathrm{~B}$ ). In addition, their pulmonary parenchyma contained impressively enlarged alveoli. These enlarged alveoli could be appreciated histologically (Fig. 5 C) and in the morphometric evaluations as significantly increased chord lengths (Fig. $5 \mathrm{H}$ ). Respiratory abnormalities were not 
seen in the lungs of transgene negative animals receiving normal water or dox water or dual transgene positive animals receiving normal water (Fig. 5, $D, E$, and $F$, respectively). In contrast, dual transgene positive animals that received dox water in utero and chronically for 2 mo thereafter manifest peribronchiolar mononuclear cell predominant nodules, subepithelial fibrosis, and large alveoli documented histologically and via morphometric analysis (Fig. 5, $G$ and $H$ ). Thus, the histologic abnormalities seen in the CC10-IL-11 transgenic mice can be reproduced in the CC10-rtTA-hIL-11 transgenic mice when the timing of gene activation in the traditional transgenic mice is reproduced with the externally regulatable system.

Importance of timing and duration of gene expression in determining pulmonary phenotype. To further define the importance of the timing and duration of gene expression in the generation of these pulmonary phenotypes, we compared the lungs of CC10-rtTA-hIL-11 mice in which IL-11 gene expression was activated at different times and for brief periods or extended intervals. When the IL-11 transgene was activated in normal mature ( $>1$-mo-old) lungs, interesting results were noted. Short periods of dox administration (2-5 d) did not consistently alter pulmonary phenotype. Occasional mononuclear cell predominant nodular changes were noted, but this was not a consistent finding (data not shown). However, extended periods of dox administration caused peribronchiolar nodules and subepithelial fibrosis (Fig. 6, $D$ and $E$, respectively). In spite of this extended period of IL-11 production, significant alveolar enlargement could not be appreciated histologically (Fig. $6 F$ ) or with morphometric techniques (Fig. $6 \mathrm{G}$ ). In contrast, IL-11 gene activation that was initiated in utero consistently led to murine lungs with large alveoli. Activation of IL-11 in utero and for 10-14 d after birth resulted in very large alveolar air spaces and impressive alterations in lung morphometry (Fig. 7, $D$ and $E$, respectively). These animals had only a small number of true alveoli and instead had lungs that were arrested in the early alveolarization phase of lung development (33). However, these animals did not manifest peribronchiolar nodules or significant subepithelial fibrosis (Fig. $7 \mathrm{D}$ ). Thus,
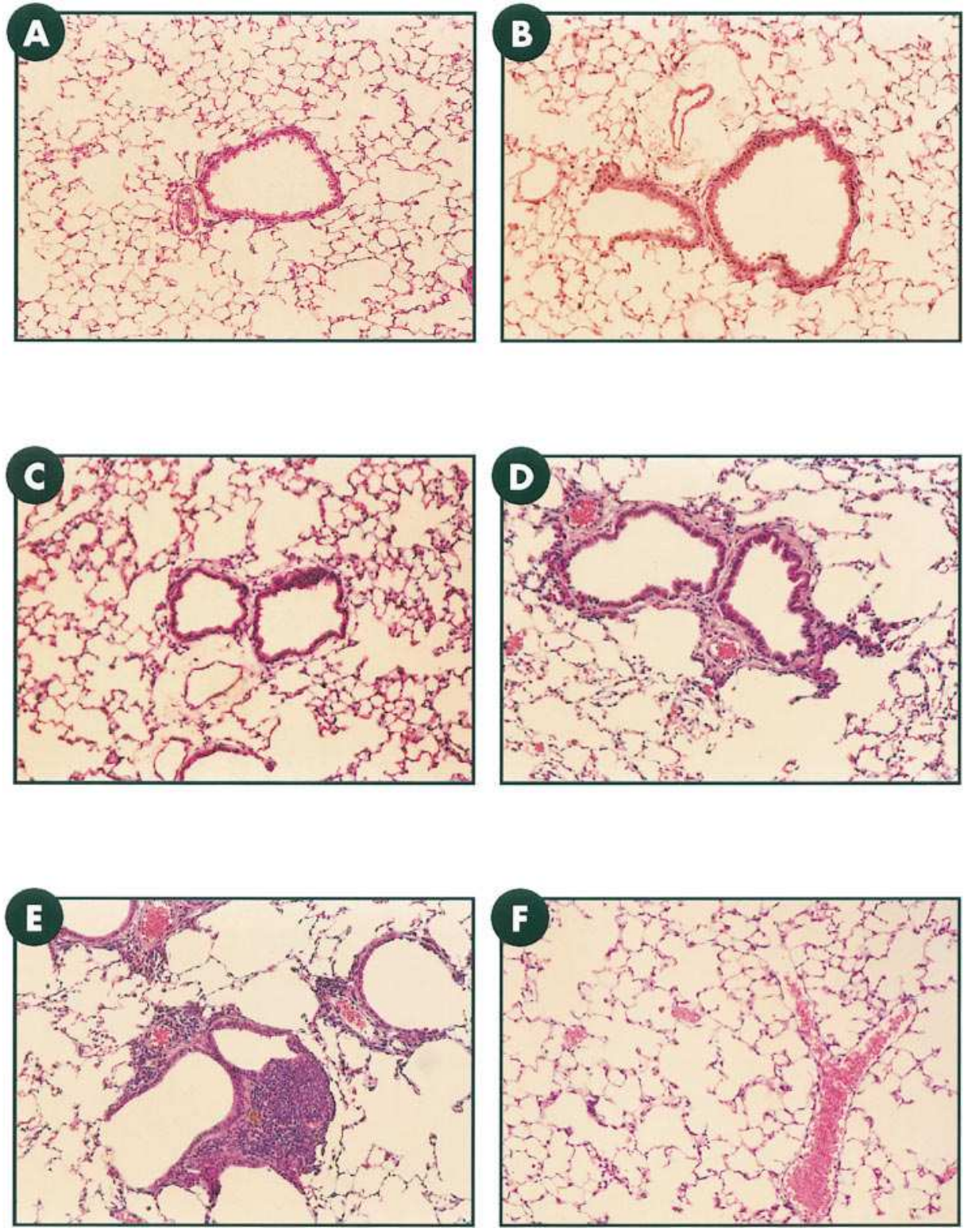

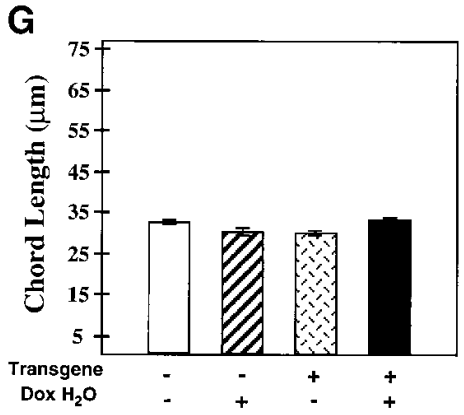

Figure 6. Airway and alveolar phenotype of CC10-rtTA-hIL-11 mice when the transgene is activated in adult animals. CC10-rtTA-hIL-11 mice and transgene negative littermate controls were maintained on normal water until they were 2 mo old. They were then given either normal water or dox water for an additional $3 \mathrm{mo}$. The normal histology of transgene negative mice receiving normal water and dox water during the 3-mo interval can be seen in $A$ and $B$, respectively. The normal histology of dual transgene positive mice that received normal water throughout the 5-mo interval can be seen in C. D illustrates the subepithelial airway fibrosis and $E$ illustrates the peribronchiolar nodules seen in dual transgene positive animals receiving dox water during the final 3 mo of the study period. $F$ illustrates the normal alveolar structure of dual transgene positive animals receiving dox water from 2 to 5 mo of age. $G$ demonstrates that dual transgene positive CC10-rtTA-hIL-11 mice that received normal water or dox water during the 3-mo interval had similar values for chord length obtained by morphometric analysis. Original magnification for all photomicrographs, $\times 39.2$. 

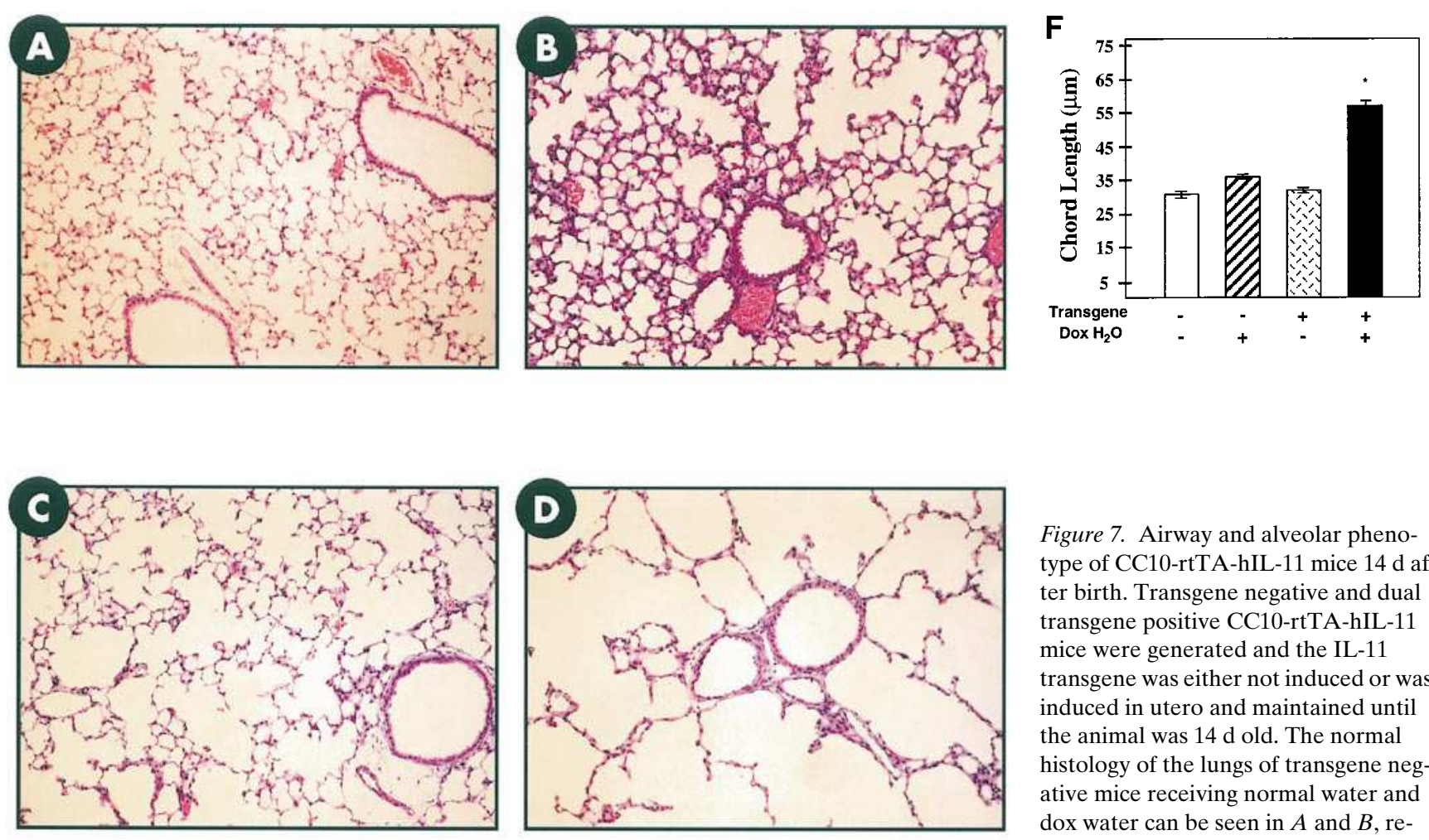

Figure 7. Airway and alveolar phenotype of CC10-rtTA-hIL-11 mice $14 \mathrm{~d}$ after birth. Transgene negative and dual transgene positive CC10-rtTA-hIL-11 mice were generated and the IL-11 transgene was either not induced or was induced in utero and maintained until the animal was $14 \mathrm{~d}$ old. The normal histology of the lungs of transgene negative mice receiving normal water and dox water can be seen in $A$ and $B$, respectively. $C$ illustrates the normal histology of the lungs of dual transgene positive mice on normal water. $D$ and $E$ demonstrate that IL-11 activation dur-

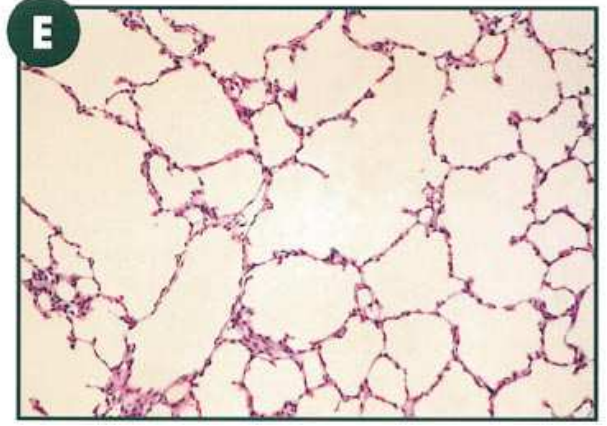
ing this time interval causes alveolar enlargement ( $D$ and $E$ ) without causing airway fibrosis $(D) . F$ illustrates the chord lengths in the alveolar portions of the lungs from the dual transgene positive animals on dox water compared with the other transgenic groups. $* P<$ 0.01 as versus transgene negative littermate mice on normal water or dox water or transgene positive littermate mice on normal water. Original magnification of all photomicrographs, $\times 39.2$.

the peribronchiolar nodules and subepithelial fibrosis seen in CC10-driven IL-11 overexpression transgenic mice occur when gene activation is initiated in utero or in adult animals and maintained for an extended period of time. In contrast, the alveolar enlargement was the result of alveolar hypoplasia and was only noted when IL-11 was activated in utero and/or in the early neonatal period. These studies demonstrate that the pulmonary phenotype of CC10-IL-11 overexpression animals contains abnormalities that are dependent on (large alveoli) and independent of (airway remodeling, peribronchiolar nodules) pulmonary growth and development and highlight the ability of the CC10-rtTA system to differentiate among these features.

\section{Discussion}

Mouse lung development begins $9.5 \mathrm{~d}$ pc with an outgrowth of paired lung buds from the foregut endoderm. This is followed by a defined series of developmental steps that form conduct- ing airways and terminal acinar buds and dilatation of the terminal lung buds to form the sac-like primary saccules that are the gas exchanging structures present at birth $(21,33)$. During the first 2-3 d of postnatal life the primary saccules enlarge and the previously smooth saccule wall is modified by the development of low "secondary crests" $(33,34)$. The primary saccules then become subdivided into alveoli by the rapid elongation of the secondary crests, a process that occurs, in great extent, between days 4 and 14 after birth (the alveolarization phase of lung development) $(33,34)$. To determine if transgene-induced alterations in this developmental format contribute to the phenotype of overexpression transgenic animals, we compared the phenotype of animals in which IL-11 was expressed under the influence of the $\mathrm{CC} 10$ promoter in the presence and absence of a genetic regulatory feature that allowed gene expression to be induced in utero or delayed until adulthood. These studies demonstrate that animals in which IL-11 was expressed in utero, and for a significant period of 
time thereafter, manifest airway remodeling, peribronchiolar nodules, and alveolar air space enlargement (emphysema). They also demonstrate that animals in which IL-11 gene expression is limited to the adult lung manifest airway remodeling and nodules without emphysema and that emphysema alone was seen when gene expression was activated in utero and stopped within $14 \mathrm{~d}$ of birth. Thus, the ability of IL-11 to cause airway remodeling and peribronchiolar inflammatory nodules appears to be independent of pulmonary growth and development. In contrast, the alveolar enlargement that is seen in IL-11 overexpression transgenic animals represents alveolar hypoplasia due to a defect in alveolarization of the developing lung. These studies are the first to demonstrate that IL-11 can alter pulmonary alveolar development. Importantly, they are also the first to demonstrate that conventional overexpression transgenic animals can have complex phenotypes that include features that are dependent and independent of lung growth and development and that inducible transgenic systems, such as the one described in this report, can be used to differentiate among these manifestations.

Numerous reports have demonstrated that the stage of alveolarization is a very critical period of lung development that is highly sensitive to a variety of influences. Glucocorticoids, hypoxia, hyperoxia, and inhibitors of thyroid function all impede normal alveolar development $(33,35)$. The present studies further support this contention by demonstrating that the overexpression of IL-11 during this developmental stage also inhibits the formation of alveoli. These observations have important implications for a number of other transgenic systems that have been reported to date. Large alveoli (emphysema) were noted in overexpression transgenic mice in which the surfactant apoprotein-C promoter was used to drive the expression of TNF- $\alpha$ (19)and TGF- $\alpha$ (18). In the TNF- $\alpha$ overexpression mice, it was assumed that the alveolar enlargement was the result of the rupture of alveolar septa. However, a cytokine-induced defect in alveolar development, analogous to that described in this report is an attractive alternative explanation. Transgenic modeling with an inducible system might be useful in addressing the mechanism of alveolar enlargement in these animals.

The mechanism by which IL-11 causes alveolar hypoplasia is unknown. However, there are a number of clues that can be derived from the biology of IL-11 and the known mechanisms of alveolus formation. First, $\alpha$-smooth muscle actin-containing cells (presumably myocytes and myofibroblasts) have been shown to play an important role in lung morphogenesis, possibly by providing a mesenchymal influence that stabilizes the lung's branching points allowing the expansion of the distal leading edges of epithelial tubules and terminal buds $(21,36)$. The homozygous knockout of the platelet-derived growth factor- $\alpha$ gene (36) and the overexpression of TGF- $\beta_{1}(21)$, which eliminate and increase the number of $\alpha$-smooth muscle actin positive cells, respectively, both lead to defects in alveolar development. Previous studies from our laboratory demonstrated that IL-11 induces the development of $\alpha$-smooth muscle actincontaining cells in airway structures (16). Thus, it is tempting to hypothesize that IL-11 might be mediating its effects on alveolar development via stimulating $\alpha$-smooth muscle actin cell accumulation. However, if abnormalities in $\alpha$-smooth muscle actin-containing cells are noted, it is also possible that they are the result of, and not the cause of, the IL-11-induced developmental abnormalities. In addition, since TGF- $\beta_{1}$ is an impor- tant stimulator of IL-11 production by a variety of stromal tissues $(23,24)$ it is reasonable to hypothesize that the effects of TGF- $\beta_{1}$ may be mediated, in part, by IL-11 in these overexpression modeling systems. An additional interesting property of IL-11 is its ability to inhibit epithelial cell proliferation (37). Since epithelial proliferation is required for the formation and elongation of structures like alveolar septa (35), it is easy to see how an inhibitor of epithelial proliferation might alter the development of alveolar structures. Additional investigations will be needed to differentiate among these and other possible mechanisms of this intriguing biologic event.

Inducible transgenic systems such as the one described in this report are most useful when the basal levels of transgene expression are below the levels at which biologic effects of the transgene can be seen and the induced levels of the transgene are well within the physiologic range of the expressed protein. An analysis of the CC10-rtTA-hIL-11 mice suggests that this has been accomplished in our system. In the absence of dox, BAL levels of IL-11 $\leq 50 \mathrm{pg} / \mathrm{ml}$ were noted and histologic alterations in pulmonary structure were not appreciated. This observation is in accord with a number of studies that demonstrate that IL- 11 concentrations $>50 \mathrm{pg} / \mathrm{ml}$ are required to induce important IL-11 biologic effects in vitro (38-40). In contrast, dual transgene positive animals receiving dox water had levels of BAL IL- $11 \geq 0.3 \mathrm{ng} / \mathrm{ml}$ and major alterations in their airway and alveolar structures. This is in keeping with studies in the literature demonstrating that nanogram per milliliter concentrations of IL-11 are found in supernatants from appropriately stimulated stromal cells in vitro $(23,24,26)$ and that picogram per milliliter to nanogram per milliliter concentrations of IL-11 are present in the nasal washings of children experiencing upper respiratory tract infections (26). However, it is important to view these conclusions with caution for a number of reasons. First, it is impossible to know what the levels of IL-11 are at local tissue sites in dual transgene positive animals receiving normal water since dilution occurs during BAL. Second, IL-11 may mediate its effects, in selected circumstances, at very low concentrations. This is seen in the bioassays that are used to detect IL-11 that are based on the proliferation of specially selected plasmacytoma cells (41). Thus, although the present system appears to meet the requirements one would wish to have for an inducible transgenic model, the potential limitations of the system must always be kept in mind.

Although the timing of developmental stages and their relationship to birth can differ from one species to another, in general, the major steps and mechanisms of lung development are well preserved from species to species. Thus, insights obtained in experimental animals may be extrapolated with caution to other species (33). In keeping with these similarities, alveolar development in humans begins at week 36 of gestation and lasts into early childhood with $85 \%$ of human alveoli formed after birth (33). Not surprisingly, abnormalities of alveolar development have been reported in a number of pediatric respiratory disorders. Among the most common is bronchopulmonary dysplasia in which large alveoli can be readily appreciated (42). The mechanisms responsible for these abnormalities have not been defined. However, modeling systems such as the one described in this report offer, for the first time, an approach that can be used to characterize the processes that generate these important pathologic abnormalities.

Early events in childhood are felt to play a key role in the pathogenesis of a variety of pulmonary disorders. This is par- 
ticularly true in asthma where associations between severe viral respiratory tract infections in early life, atopy, and the development of chronic asthma have been noted (12-14). Experimental modeling of viral effects in the airway have demonstrated that temporal windows exist in which viral infections have different manifestations. For example, parainfluenza type 1 virus caused a necrotizing bronchiolitis and interstitial pneumonia in neonatal and weaning rats. However, the virus also caused airway remodeling, peribronchiolar mononuclear nodules, and alveolar hypoplasia only in neonatal rats (43). Studies of this sort demonstrate that timing is a crucial variable that influences the phenotype that arises from interactions between environmental and inflammatory stimuli and endogenous developmental programs. The regulatable overexpression transgenic system described in this report provides, for the first time, a system in which these crucial interactions can be investigated.

In summary, these studies describe the first externally regulatable organ-specific overexpression transgenic system and the utilization of this system to differentiate the developmentdependent and -independent phenotypic features caused by the overexpression of IL-11 in the murine lung. Organ-specific externally regulatable transgenic systems of this sort can provide impressive insights into the biology of protein effector functions, the biology of organ growth and development, and the timing of crucial interactions between environmental stimuli and endogenous programs of growth and development. This approach will allow us to model developmental, early life, and waxing and waning biologic processes with a level of fidelity not previously available with standard overexpression transgenic approaches.

\section{Acknowledgments}

The authors thank the investigators and institutions that provided the reagents that were used, Dr. Jeffrey A. Whitsett and Dr. Barry Stripp for the gift of the CC10 promoter, and Ms. Kathleen Bertier for her excellent secretarial assistance.

This work was supported by National Institutes of Health grants HL-36708, AI-34953, HL-54989 (to J.A. Elias), HL-56389 (to J.A. Elias, R. Homer, and R.A. Flavell), and HL-52014 (to P. Ray). R.A. Flavell is an Investigator of the Howard Hughes Medical Institute.

\section{References}

1. Mader, S., and J.H. White. 1993. A steroid-inducible promoter for the controlled overexpression of cloned genes in eukaryotic cells. Biochemistry. 90: 5603-5607.

2. Palmiter, R.D., H.Y. Chen, and R.L. Brinster. 1982. Differential regulation of metallothionein-thymidine kinase fusion genes in transgenic mice and their offspring. Cell. 29:701-710.

3. Gossen, M., and H. Bujard. 1992. Tight control of gene expression in mammalian cells by tetracycline-responsive promoters. Proc. Natl. Acad. Sci. USA. 89:5547-5551.

4. Gossen, M., A.L. Bonin, and H. Bujard. 1993. Control of gene activity in higher eukaryotic cells by prokaryotic regulatory elements. Trends Biochem. Sci. 18:471-475.

5. Gossen, M., S. Freundlieb, G. Bender, G. Muller, W. Hillen, and H. Bujard. 1995. Transcriptional activation by tetracyclines in mammalian cells. Science. 268:1766-1769.

6. Fishman, G.I., M.L. Kaplan, and P.M. Buttrick. 1994. Tetracycline-regulated cardiac gene expression in vivo. J. Clin. Invest. 93:1864-1868.

7. Kitamura, M. 1996. Creation of a reversible on/off system for site-specific in vivo control of exogenous gene activity in the renal glomerulus. Proc. Natl. Acad. Sci. USA. 98:7387-7391.

8. Kistner, A., M. Gossen, F. Zimmermann, J. Jerecic, C. Ullmer, H. Lubbert, and H. Bujard. 1996. Doxycycline-mediated quantitative and tissue-spe- cific control of gene expression in transgenic mice. Proc. Natl. Acad. Sci. USA. 93:10933-10938.

9. Efrat, S., D. Fusco-DeMane, H. Lemberg, O.A. Emran, and X. Wang. 1995. Conditional transformation of a pancreatic $\beta$-cell line derived from transgenic mice expressing a tetracycline-regulated oncogene. Proc. Natl. Acad. Sci. USA. 92:3576-3580.

10. Furth, P.A., L. St. Onge, H. Boger, P. Gruss, M. Gossen, A. Kistner, H. Bujard, and L. Hennighausen. 1994. Temporal control of gene expression in transgenic mice by a tetracycline-responsive promoter. Proc. Natl. Acad. Sci. USA. 91:9302-9306.

11. Mayford, M., M.E. Bach, Y.-Y. Huang, L. Wang, R.D. Hawkins, and E.R. Kandel. 1996. Control of memory formation through regulated expression of a CaMKII transgene. Science. 274:1678-1683.

12. Sigurs, N., R. Bjarnason, F. Sigurbergsson, B. Kjellman, and B. Bjorksten. 1995. Asthma and immunoglobulin E antibodies after respiratory syncytial virus bronchiolitis: a prospective cohort study with matched controls. Pediatrics. 95:500-505.

13. Geppert, E.F., L.A. Lester, and C. Ober. 1995. Prioritizing asthma research: the need to investigate childhood asthma. Am. J. Respir. Crit. Care Med. 151:1294-1295.

14. Busse, W., S.P. Banks-Schlegel, and G.L. Larsen. 1995. NHLBI Workshop Summary: Childhood- versus adult-onset asthma. Am. J. Respir. Crit. Care Med. 151:1635-1639.

15. Rankin, J.A., D.E. Picarella, G.P. Geba, U.-A. Temann, B. Prasad, B. DiCosmo, A. Tarallo, B. Stripp, J. Whitsett, and R.A. Flavell. 1996. Phenotypic and physiologic characterization of transgenic mice expressing interleukin 4 in the lung: lymphocytic and eosinophilic inflammation without airway hyperreactivity. Proc. Natl. Acad. Sci. USA. 93:7821-7825.

16. Tang, W., G.P. Geba, T. Zheng, P. Ray, R. Homer, C.I. Kuhn, R.A. Favell, and J.A. Elias. 1996. Targeted expression of IL-11 in the murine airway causes airways obstruction, bronchial remodeling and lymphocytic inflammation. J. Clin. Invest. 98:2845-2853.

17. DiCosmo, B.F., G.P. Geba, D. Picarella, J.A. Elias, J.A. Rankin, B.R Stripp, J.A. Whitsett, and R.A. Flavell. 1994. Airway targeted interleukin 6 in transgenic mice. Uncoupling of airway inflammation and bronchial hyperreactivity. J. Clin. Invest. 94:2028-2035.

18. Korfhagen, T.R., R.J. Swantz, S.E. Wert, J.M. McCarty, C.B. Kerlakian, S.W. Glasser, and J.A. Whitsett. 1994. Respiratory epithelial cell expression of human transforming growth factor- $\alpha$ induces lung fibrosis in transgenic mice. $J$. Clin. Invest. 93:1691-1694.

19. Miyazaki, Y., K. Araki, C. Vesin, I. Garcia, Y. Kapanci, J.A. Whitsett, P.-F. Piguet, and P. Vassalli. 1995. Expression of a tumor necrosis factor- $\alpha$ transgene in murine lung causes lymphocytic and fibrosing alveolitis. J. Clin. Invest. 96:250-259.

20. Ho, Y.-S. 1994. Transgenic models for the study of lung biology and disease. Am. J. Physiol. (Lung Cell. Mol. Physiol. 10). 266:L319-L353.

21. Zhou, L., C.R. Dey, S.E. Wert, and J.A. Whitsett. 1996. Arrested lung morphogenesis in transgenic mice bearing an SP-C-TGF- $\beta 1$ chimeric gene. Dev. Biol. 175:227-238.

22. Zheng, T., M. Nathanson, and J.A. Elias. 1994. Histamine augments cytokine-stimulated interleukin-11 production by human lung fibroblasts. J. Immunol. 153:4742-4752.

23. Elias, J.A., T. Zheng, N.L. Whiting, T.K. Trow, W.W. Merrill, R. Zitnik, P. Ray, and E.M. Alderman. 1994. Interleukin-1 and transforming growth factor $\beta$ regulation of fibroblast-derived interleukin-11. J. Immunol. 152:24212429.

24. Elias, J.A., T. Zheng, O. Einarsson, M. Landry, T.K. Trow, N. Rebert, and J. Panuska. 1994. Epithelial interleukin-11: regulation by cytokines, respiratory syncytial virus and retinoic acid. J. Biol. Chem. 169:22261-22268.

25. Rochester, C.L., S.J. Ackerman, T. Zheng, and J.A. Elias. 1996. Eosinophil-fibroblast interactions: granule major basic protein stimulation of fibroblast interleukin-6, interleukin-11 and leukemia inhibitory factor expression. $J$. Immunol. 156:4449-4456.

26. Einarsson, O., G.P. Geba, Z. Zhu, M. Landry, and J.A. Elias. 1996. Interleukin 11: stimulation in vivo and in vitro by respiratory viruses and induction of airways hyperresponsiveness. J. Clin. Invest. 97:915-924.

27. Pack, R.J., L.H. Al-Ugaily, and G. Morris. 1981. The cells of the tracheo-bronchial epithelium of the mouse: a quantitative light and electron microscope study. J. Anat. 132:71-84.

28. Stripp, B.R., P.L. Sawaya, D.S. Luse, K.A. Wikenheiser, S.E. Wert, J.A Huffman, D.L. Lattier, G. Singh, S.L. Katyal, and J.A. Whitsett. 1992. Cis-acting elements that confer lung epithelial cell expression of the CC10 gene. $J$. Biol. Chem. 267:14703-14712.

29. Gross, I., and C.M. Wilson. 1983. Fetal rat lung maturation: initiation and modulation. J. Appl. Physiol. (Respirat. Environ. Exercise Physiol.) 55: 1725-1732.

30. Escolar, J.D.D., B. Gallego, C. Tejero, and M.A. Escolar. 1994. Changes occurring with increasing age in the rat lung: morphometrical study. Anat. Rec. 239:287-296.

31. Weibel, E.R. 1979. Stereological methods. In Practical Methods for Biological Morphometry. Vol. 1. Academic Press, Inc., London. 30.

32. Gundersen, H.J.G., and R. Osterby. 1981. Optimizing sampling effi- 
ciency of stereological studies in biology: or "Do more less well!". J. Microsc. 121:65-73.

33. Burri, P.H. 1997. Structural aspects of prenatal and postnatal development and growth of the lung. In Lung Growth and Development. Vol. 100. J.A. McDonald, editor. Marcel Dekker, Inc., New York. 1-35.

34. Amy, R.W., D. Bowes, P.H. Burri, J. Haines, and W.M. Thurlbeck. 1977. Postnatal growth of the mouse lung. J. Anat. 124:131-151.

35. Massaro, D., N. Teich, S. Maxwell, G.D. Massaro, and P. Whitney. 1985. Postnatal development of alveoli. J. Clin. Invest. 76:1297-1305.

36. Bostrom, H., K. Willetts, M. Pekny, P. Leveen, P. Lindahl, H. Hedstrand, M. Pekna, M. Hellstrom, S. Gebre-Medhin, M. Schalling, et al. 1996. PDGF-A signaling is a critical event in lung alveolar myofibroblast development and alveogenesis. Cell. 85:863-873.

37. Peterson, R.L., M.M. Bozza, and A.J. Dorner. 1996. Interleukin-11 induces intestinal epithelial cell growth arrest through effects on retinoblastoma protein phosphorylation. Am. J. Pathol. 149:895-902.

38. Leng, S.X., and J.A. Elias. 1997. Interleukin-11 inhibits macrophage interleukin-12 production. J. Immunol. 159:2161-2168.
39. Redlich, A.A., X. Gao, S. Rockwell, M. Kelley, and J.A. Elias. 1996. IL11 enhances survival and decreases TNF production after radiation-induced thoracic injury. J. Immunol. 157:1705-1710.

40. Trepicchio, W.L., M. Bozza, G. Pedneault, and A.J. Dorner. 1996. Recombinant human IL-11 attenuates the inflammatory response through downregulation of proinflammatory cytokine release and nitric oxide production. $J$. Immunol. 157:3627-3634.

41. Gu, Z.-J., J. Wijdenes, X.-G. Zhang, M.-M. Hallet, C. Clement, and B. Klein. 1996. Anti-gp 130 transducer monoclonal antibodies specifically inhibiting ciliary neurotrophic factor, interleukin-6, interleukin-11, leukemia inhibitory factor or oncostatin M. J. Immunol. Methods. 190:21-27.

42. Margraf, L.R., J.F.J. Tomashefski, M.C. Bruce, and B.B. Dahms. 1991. Morphometric analysis of the lung in bronchopulmonary dysplasia. Am. Rev. Respir. Dis. 143:391-400.

43. Castleman, W.L., R.L. Sorkness, R.F. Lemanske, G. Grasee, and M.M. Suyemoto. 1988. Neonatal viral bronchiolitis and pneumonia induces bronchiolar hypoplasia and alveolar dysplasia in rats. Lab. Invest. 59:387-396. 